
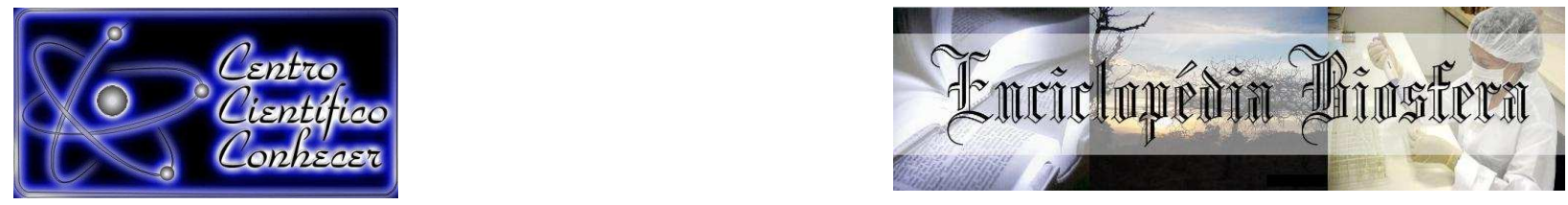

\title{
PENECTOMIA TOTAL EM Geochelone carbonaria (SPIX, 1824) - RELATO DE CASO
}

Elton Luís Ritir Oliveira1, Lívia de Lima Melo1, Edson Moura da Silva², Victor Fernando Santana Lima ${ }^{2}$, Pierre Barnabé Escodro ${ }^{3}$

1. Graduando em Medicina Veterinária, Universidade Federal de Alagoas, campus Arapiraca, Unidade de Ensino Viçosa, Viçosa, Alagoas, Brasil. elton.ritir@hotmail.com

2. Mestrando do programa de Pós-Graduação em Ciência Animal Tropical, Universidade Federal Rural de Pernambuco, Recife, Pernambuco, Brasil.

3. Prof. Adjunto da Universidade Federal de Alagoas, campus Arapiraca, Unidade de Ensino Viçosa, Viçosa, Alagoas, Brasil.

\section{Recebido em: 08/09/2015 - Aprovado em: 14/11/2015 - Publicado em: 01/12/2015} DOI: http://dx.doi.org/10.18677/Enciclopedia_Biosfera_2015_203

\begin{abstract}
RESUMO
O jabuti-piranga (Geochelone carbonaria Spix, 1824) é um testudine terrestre, pertencente à família Testudinidae, o qual encontra-se distribuído em países tropicais como o Brasil, Equador, Guianas, Paraguai, Venezuela e algumas ilhas do Caribe. No Brasil, G. carbonaria é considerada uma das principais espécies de jabutis constituintes da fauna brasileira. Neste estudo, um G. carbonária foi diagnosticado com prolapso peniano, esta alteração patológica vem sendo observada com maior frequência em clínicas veterinárias que atendem animais silvestres, provenientes de cativeiros. Muitos pesquisadores atribuem o surgimento do prolapso peniano em testudines, ao manejo inadequado aos quais estes animais são submetidos. $\mathrm{O}$ animal foi contido fisicamente e assim, procedido o exame físico, sendo observado prolapso peniano, com inflamação, escoriações e falta de retração peniana, além de alteração de cor e odor fétido. Em seguida 0 animal foi encaminhado ao centro cirúrgico para realização da penectomia. $O$ animal foi reavaliado ao $15^{\circ}$ dia pós-cirurgia, sendo relatado pelo proprietário que o jabuti estava se alimentando normalmente, além de não ter sido observado recidiva. Embora, recidivas possam ocorrer em alguns casos, o paciente deste relato obteve melhora clínica, sendo essa conduta eficaz para esta afecção.
\end{abstract}

PALAVRAS-CHAVE: jabuti-piranga, prolapso, pênis

\section{TOTAL PENECTOMY IN Geochelone carbonaria (Spix, 1842) - CASE REPORT}

\begin{abstract}
Red-footed-tortoise (Geochelone carbonaria Spix, 1824) is a terrestrial testudine, belonging to the family Testudinidae, which is distributed in tropical countries such as Brazil, Ecuador, Guyana, Paraguay, Venezuela and some Caribbean islands. In Brazil, G. carbonaria is considered one of the main species of tortoises constituents of Brazilian fauna. In this study, a G. carbonaria was diagnosed with penile prolapse, this pathological change has been observed more frequently in veterinary clinics that attend wild animals from captivity. Many researchers attribute the rise of penile prolapse in testudines, and improper management to which these animals are
\end{abstract}


subjected. The animals were physically restrained and thus carried physical examination, penile prolapse being observed with inflammation, abrasions and lack of penile retraction, and color and foul odor change. Then, the animal was taken to the operating room to perform the penectomy. The animal was valued at the 15th postoperative day, being reported by the owner that the tortoise was eating normally, besides not having been observed relapse. Although relapses may occur in some cases, the patient received this report clinical improvement, and this conduct effective for this condition.

KEYWORDS: red-footed-tortoise, prolapse, penis

\section{INTRODUÇÃO}

Os testudines são animais pertencentes à classe Reptilia, que habitam o planeta a mais de 200 milhões de anos (OBST et al., 1988). Sabe-se que jabutis, cágados e tartarugas são os principais representantes dessa classe, os quais acabaram se diferenciando das outras espécies de répteis devido à presença de um casco, o qual auxilia na proteção de predadores e de fatores ambientais (GOULART, 2004).

O jabuti-piranga (Geochelone carbonaria Spix, 1824) é um testudine terrestre, pertencente à família Testudinidae, o qual encontra-se distribuído em países tropicais como o Brasil, Equador, Guianas, Paraguai, Venezuela e algumas ilhas do Caribe (PRITCHARD, 1979; LEVINE \& SCHAFER, 1992). No Brasil o G. carbonaria é considerada uma das principais espécies de jabutis constituintes da fauna brasileira (FLOSI et al., 2000). Este testudine é conhecido por apresentar um corpo inteiramente coberto por uma carapaça superior e um plastrão na região inferior, estes são formados por placas ósseas poligonais, unidas entre si, cobertas por placas córneas negras com manchas amareladas no centro e os membros locomotores possuem manchas vermelhas (JAHN, 1990; POUG, 1997). Os machos possuem um plastrão côncavo e cauda longa, diferentemente das fêmeas, que possuem plastrão reto e cauda curta (BEYNON \& COOPER, 1994; ERNST \& BARBOUR, 1989).

Sabe-se que esta espécie de testudine vem sendo observada com maior frequência em cativeiros, os quais estão se tornando animais de estimação, devido a hábitos culturais e ao tráfico animal (PINHEIRO \& MATIAS, 2004). Quando criados em cativeiro, os jabutis necessitam de cuidados especiais, uma vez que quando submetidos a péssimas condições de manejo, acabam se tornando susceptíveis a diversas doenças e patologias (SANTOS \& PEREIRA, 2011). As enfermidades do sistema reprodutivo (p. ex.: distocia, prolapso peniano, prolapso da cloaca, cólon, bexiga e oviduto) são os principais problemas que acometem esses animais quando mantidos em cativeiro (AQUILAR et al., 2006; MATIAS et al., 2006).

O priapismo é uma afecção resultante de acidentes no momento da cópula, devido a movimentação repentina dos animais, estiramento do órgão, traumas, hiperparatireoidismo, problemas neurológicos, parasitas intestinais, cálculo vesical ou cloacal. Nesses casos, a única alternativa é a intervenção cirúrgica para amputação peniana (RAMOS et al., 2009).

Uma vez que os jabutis são testudines que vêm sendo frequentemente atendidos na clínica de animais silvestres com problemas no trato reprodutivo, o objetivo deste trabalho é relatar a realização de penectomia em um G. carbonaria (Spix, 1824). 


\section{RELATO DE CASO}

Foi atendido no serviço ambulatorial do Departamento de Medicina Veterinária da Universidade Federal de Alagoas, campus Arapiraca, Viçosa, um G. carbonaria (jabuti-piranga), macho, de 19 anos, pesando $4,3 \mathrm{~kg}$. O proprietário relatou que 0 jabuti apresentava-se com exposição do pênis e falta de apetite a cerca de quatro dias. O mesmo descreveu que o animal era mantido em um recinto com cerca de $2 \mathrm{~m}^{2}$, juntamente com outros dois jabutis fêmeas, e ambos alimentavam-se de verduras e folhas.

Feito o exame físico, foi observado prolapso peniano, com inflamação, escoriações e falta de retração peniana, além de alteração de cor e odor fétido (Figura 1). Em seguida o animal foi encaminhado ao centro cirúrgico para realização da penectomia. Inicialmente foi realizado antissepsia local com iodopirrolidona, e assim, realizado a indução anestésica com Cetamina (30 mg/kg) e Diazepam (1 $\mathrm{mg} / \mathrm{kg}$ ) via intramuscular e lidocaína (5 mg/kg) epidural intercoccígea (Cc1-Cc2) com agulha 25x07. Com o paciente na posicionado em decúbito dorsal, foi realizado antissepsia da região pericloacal e dos apêndices pélvicos. Após o miorrelaxamento e exposição completa do pênis, o qual foi posicionado sobre o plastrão, os corpos cavernosos foram transfixados individualmente com fio poliglecaprone 25 ํo 0 (Figura 2). A remoção de parte do pênis, foi realizada cerca de $1,5 \mathrm{~cm}$ proximal ao tecido desvitalizado, assegurando-se a total remoção (Figura 3). A sutura do coto peniano foi realizada em padrão único contínuo Shimidden, também com fio de poliglecaprone.

No pós-operatório, o animal recebeu enrofloxacina intramuscular $(10 \mathrm{mg} / \mathrm{kg})$ uma vez por dia, até 5 dias, sendo submetido a hidratação oral com soro glicosado $5 \%(10 \mathrm{~mL} / \mathrm{kg} / \mathrm{hora})$ até retorno de alimentação após 48 horas. O uso de suplemento vitamínico (Glicopan Pet ${ }^{\Theta}-5$ gotas ao dia) e a troca de curativo foi recomendado por 10 dias. $O$ animal foi reavaliado ao $15^{\circ}$ dia pós-cir urgia, sendo relatado pelo proprietário que o jabuti estava se alimentando normalmente.

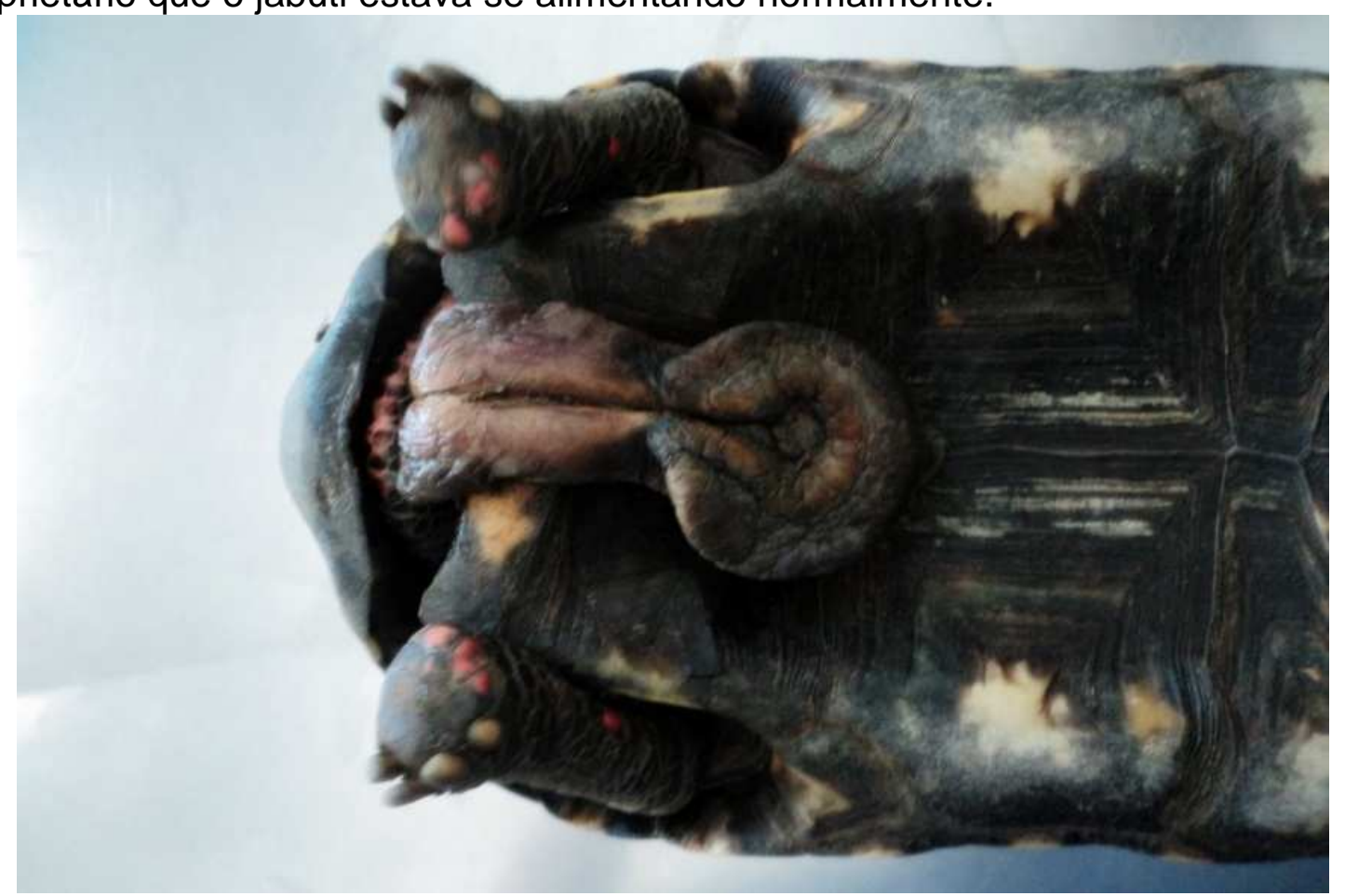

FIGURA 1. G. carbonaria apresentando pênis prolapsado.

Fonte: Arquivo pessoal (2015). 


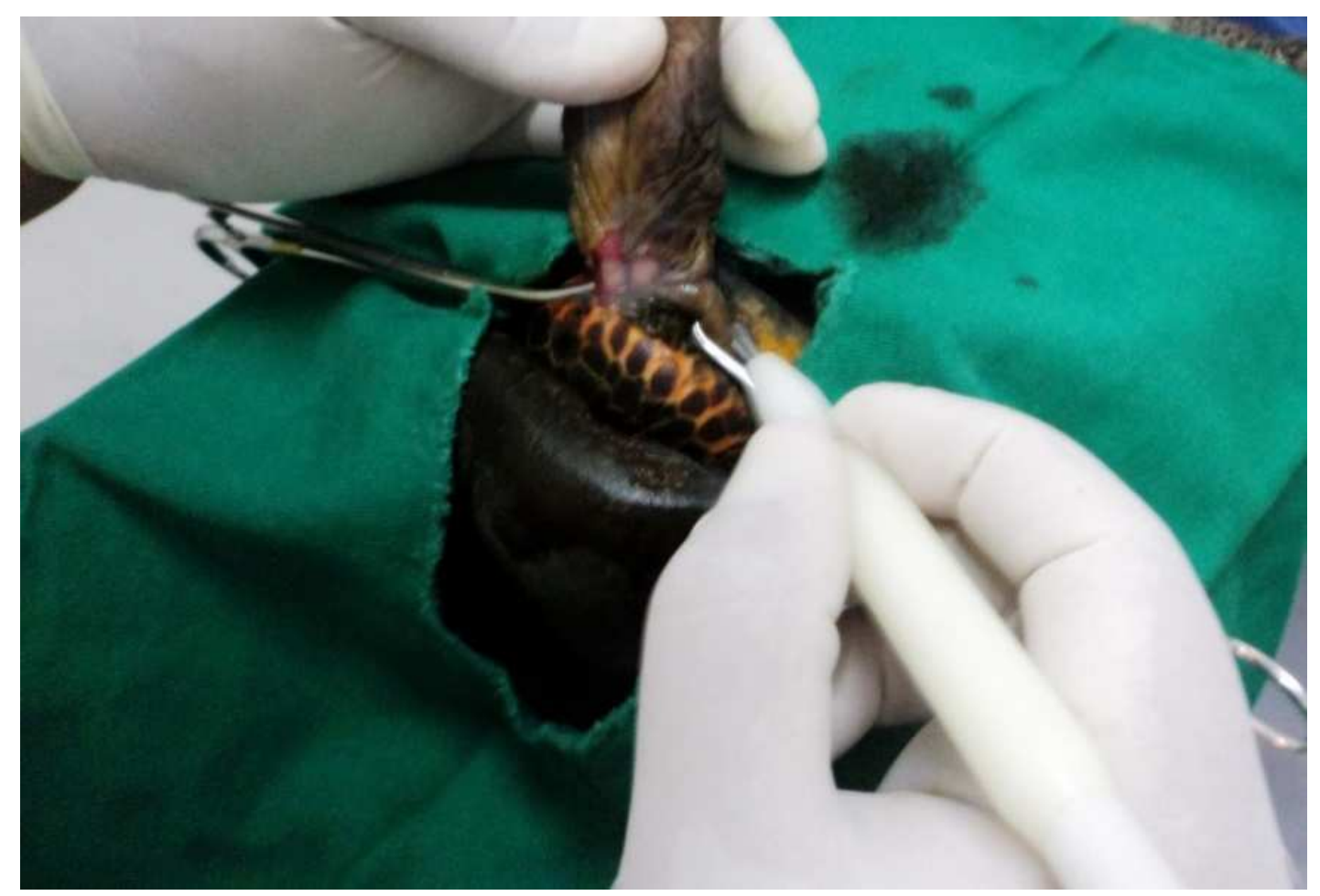

FIGURA 2. Sutura nos corpos cavernosos de pênis prolapsado de $G$. carbonaria. Fonte: Arquivo pessoal (2015).

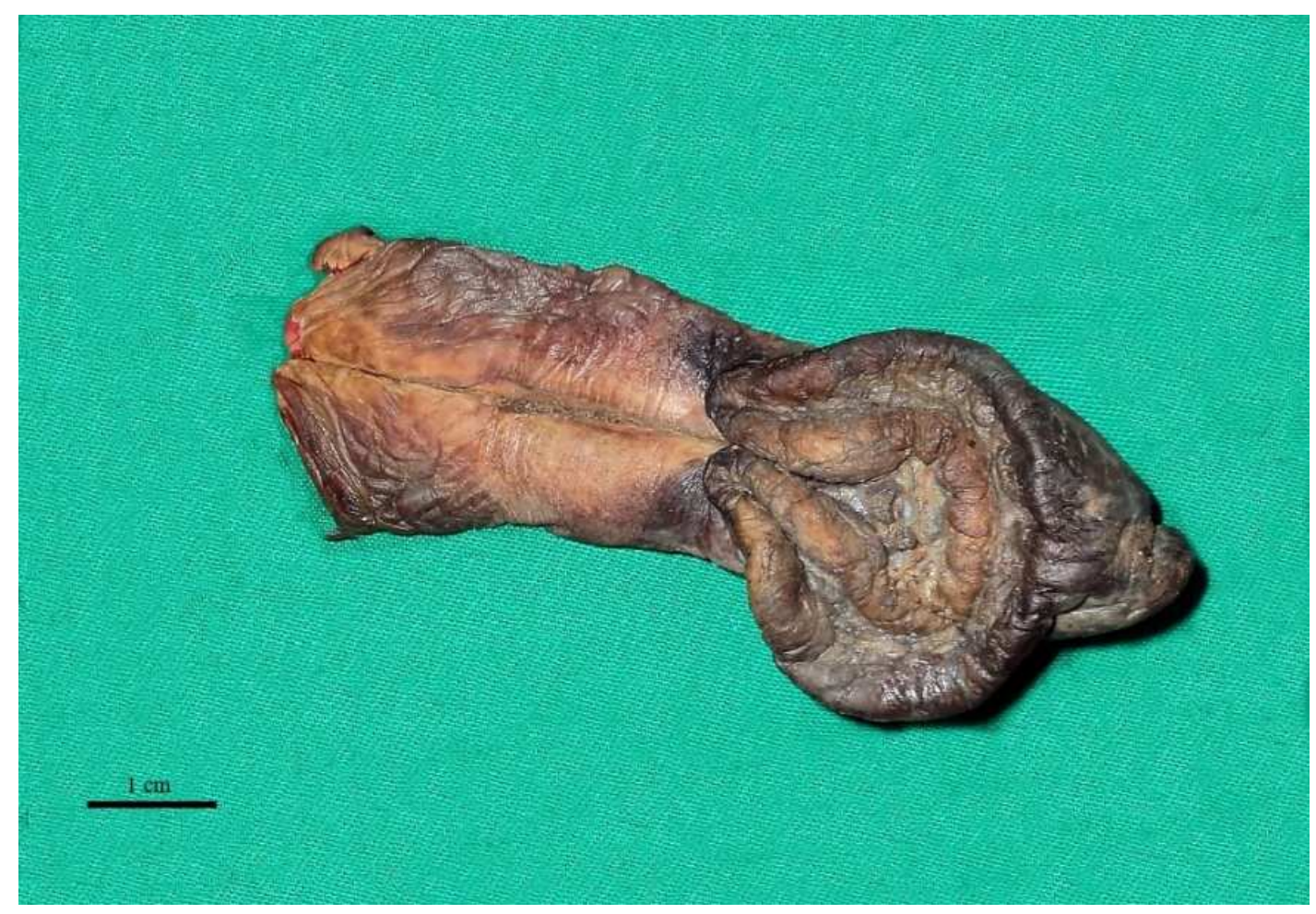

FIGURA 3. Pênis prolapsado removido de G. carbonaria. Fonte: Arquivo pessoal (2015). 


\section{RESULTADOS E DISCUSSÕES}

Neste estudo um G. carbonaria (jabuti-piranga) foi diagnosticado com prolapso peniano, esta alteração patológica vêm sendo observada com maior frequência em clínicas veterinárias que atendem animais silvestres, provenientes de cativeiro (RAMOS et al., 2009). Muitos pesquisadores, atribuem o surgimento do prolapso peniano em testudines, ao manejo inadequado aos quais estes animais são submetidos (AQUILAR et al., 2006; MATIAS et al., 2006).

Sabe-se que as exigências nutricionais dos jabutis são bastante distintas de outras espécies animais, estes repteis necessitam de uma alimentação a base de frutas, verduras e proteína animal ( $p$. ex.: peixe, minhoca e ração de carnes vermelhas) (RAMOS et al., 2009). O paciente deste relato recebia uma alimentação inadequada para a espécie, podendo este ser um dos fatores que influenciou no surgimento do prolapso peniano.

O diagnóstico de prolapso peniano no paciente deste relato foi através do exame físico, entretanto a cloacoscopia e cistoscopia são técnicas sofisticadas que vem sendo utilizado para detecção de distúrbios reprodutivos em testudines (MARTÍNEZ-SILVESTRE et al., 2015).

É importante ressaltar que o uso Cetamina, benzodiazepínicos e lidocaína como sedativos e anestésico para realização de procedimentos clínicos e cirúrgicos em jabutis é recomendado, devido ao baixo risco ou ausência de efeitos colaterais (PESSOA et al., 2009; SANTOS et al., 2009). A Cetamina permitiu uma boa sedação, mantendo os movimentos voluntários das vias aéreas (HARMS et al., 2014), já o diazepam por via intramuscular e a lidocaína aplicada por via epidural nas vertebras coccídeas, proporcionaram o total relaxamento da cauda, pênis e membros pélvicos (ALVES JÚNIOR et al., 2012).

Embora, recidivas possam ocorrer em alguns casos, o paciente deste relato obteve melhora clínica, sendo essa conduta eficaz para esta afecção. Uma vez que, o uso de sutura com fio pologlecaprone e padrão Shimidden possibilitou menor tempo cirúrgico, não havendo a necessidade de retirada de pontos, diminuindo possível estresse do animal (RIBEIRO et al., 2005). A remoção total do pênis em testudines pode ser realizada, não tendo qualquer alteração envolvendo o sistema urinário, pois a uretra destes animais elimina urina no canal cloacal (MADER, 2006).

\section{CONCLUSÃO}

Conclui-se que a penectomia total é um procedimento eficaz em casos de prolapso peniano com necrose tecidual, pois evita que 0 animal venha ater recindiva, porém vale ressaltar que este procedimento torna-o incapaz de ter uma vida reprodutiva.

\section{REFERÊNCIAS}

ALVES JÚNIOR, J.R.; BOSSO, A.C.; ANDRADE, M.B.; WERTHER, K.; SANTOS A.L. Association of midazolam with ketamine in giant Amazon river turtles Podocnemis expansa breed in captivity. Acta Cirúrgica Brasileira, v. 27, n.2, p.144147, 2012.

AQUILAR R.; DIVERS S.M.H.; DIVERS S.J.H. Atlas de Medicina Terapêutica e Patologia de Animais Exóticos. São Caetano do Sul: Interbook, p. 375, 2006.

BEYNON, P.H.; COOPER, J.E. Manual of exotic pets. Barcelona: British Small Animal Veterinay Association, p. 224, 1994. 
ERNEST, C.H.; BARBOUR, R.W. Turtles of the world. Washington: Smithsonian Institution Press, p. 253-254, 1989.

FLOSSI, F. M; GARCIA, J. M; PUGLIESE, C; SANCHEZ, A.A; KLAI, A. Manejo e enfermidades de quelônios brasileiros no cativeiro doméstico. Revista de Educação Continuada. São Paulo, vol.4, fascículo 2, p. 65-72, 2001.

GOULART, C. E. S. Herpetologia, herpetocultura e medicina de répteis. Rio de Janeiro: L.F. Livros de Veterinária, p. 45, 2004.

HARMS, C.A.; PINIAK, W.E.; ECKERT, S.A.; STRINGER, E.M. Sedation and anesthesia of hatchling leatherback sea turtles (Dermochelys coriacea) for auditory evoked potential measurement in air and in water. The Journal of Zoo and Wildife Medicine, v. 45, n.1, p. 86-92, 2014.

JAHN, K. Mis Tortugas. Barcelona: Editorial Hispano Europea, p.48, 1990.

LEVINE, D.; SCHAFER, D. Tortuga Gaz., v.28, p.1-3, 1992.

MARTÍNEZ-SILVESTRE, A., BARGALLÓ, F., GRÍFOLS, J. Gender Identification by Cloacoscopy and Cystoscopy in Juvenile Chelonians. Veterinary Clinics of North America: Exotic Animal Practice, v.8, p.527-539, 2015.doi:10.1016/j.cvex.2015.04.009.

MATIAS, C.A. $R$ et al. Aspectos fisiopatológicos da retenção de ovos em Jabutipiranga (Geochelone carbonária, Spix, 1824). Ciência Rural, v. 36, p.1494 1500, 2006.

OBST, F.J.; RICHTER, K.; JACOB, U. The completely illustrated atlas of reptile and amphibians for the terrarium. New Jersey: TFH, 1988. 831p.

PESSOA, C.A.; PRAZERES, R.F.; FECCHIO, R.S.; RODRIGUES, M.A.; SOUZA, P.C. Utilização da videoendoscopia no diagnóstico de balanite em Jabuti-Piranga (Geochelone carbonaria Spix, 1824) - Relato de caso. In: XII CONGRESSO E XVIII ENCONTRO DA ASSOCIAÇÃO BRASILEIRA DE VETERINÁRIOS DE ANIMAIS SELVAGENS. Anais..Águas de Lindóia, São Paulo, 2009.

PINHEIRO FP, MATIAS CAR (2004). Fauna silvestre apreendida e resgatada no estado do Rio e Janeiro. In: Congresso Da Sociedade de Zoológicos do Brasil, Rio de Janeiro. Anais..., 28,2003.

POUGH, A.S. Anatomia comparativa dos vertebrados. São Paulo: Atheneu, 1985, p. 322-337.

PRITCHARD, P.C.H. Encyclopedia of turtles. New Jersy: TFD, 1979. p.326-323.

RAMOS, R. M.; VALE, D. F. V.; HANAWO, M. E. O. C. FERREIRA, F. S.; UZ, M. J.; OLIVEIRA, A. L. A. Penectomia em caso de prolapso peniano em Jabuti-piranga (Geochelone carbonaria) - Relato de caso. Jornal Brasileiro de Ciência Animal, v.2, n.3, p.166-174, 2009.

RIBEIRO, C.; SILVA-JÚNIOR, VA.; SILVA-NETO, J.C.; VASCONCELOS, B.C. Clinical and histopathological study of tissue reactivity to monofilament suture materials: nylon and poliglecaprone 25 in rats. Acta Cirúrgica Brasileira, v.20, n,4, p.284-91, 2005. 
SANTOS, A.L.Q.; HIRANO, L.Q.L.; PEREIRA, P.C.; SILVA, J.M.M.; KAMINISHI, A.P.S.; BOSSO, A.C.S. Anestesia de cagado-de-barbicha Phrynops geoffroanus Schweigger de 1812 (Testudines) com um e midazolan Associação propofol. Acta Scientiarum, v. 31, n.3, p. 317-21, 2009.

SANTOS, G. J.; PEREIRA, R. E. P. Amento de Aspergillus fumigatus e Strongyloides sp. em jabutis mantidos em cativeiro no Bosque Municipal Dr. Belírio Guimarães Brandão- Zoologico Municipal da cidade de Garça- SP. Revista Científica Eletrônica de Medicina Veterinária. Graça, ano IX, n.16, p. 01-29, 2011. 